\title{
Association Between Expression of Intercellular Adhesion Molecule-1 and Integration of Human T-cell-leukemia Virus Type 1 in Adult T-cell Leukemia Cells

\author{
Satoshi Motoya, Masayuki Tsujisaki, ${ }^{*}$ Tsuneharu Jinnohara, Shigeru Sasaki, \\ Tatsumi Nakano, Yuji Hinoda, and Kohzoh Imai
} \\ First Department of Internal Medicine, Sapporo Medical University School of Medicine, Sapporo, Japan
}

It is known that the expression levels of intercellular adhesion molecule-1 (ICAM-1) in adult $\mathrm{T}$ cell leukemia(ATL) cells are high, whereas those in T-lymphoid cells are not. In order to investigate the factors that influence the induction of ICAM-1 molecules, Northern blot analysis to measure the expression level of ICAM-1 mRNAs and Southern blot hybridization to analyze the integration of human Tcell-leukemia virus type 1 (HTLV-1) provirus were done. The levels of ICAM-1 mRNA expression of ATL cells were generally higher than those of T-lymphoid cells. However, ILT- mat cells and ATL16T(-) cells, although they were ATL cells, showed rather low surface ICAM-1 expression and ICAM-1 mRNA expression. Southern blot hybridization showed that only two and four bands were found in ILT-mat and ATL16T(-) cells, respectively, whereas $>10$ bands were detected in other ATL cells. These results suggest that monoclonal integration of HTLV-1 provirus to the genome of $\mathrm{T}$ cell, especially the number of integration sites, is one of the factors for induction of ICAM-1 molecules. J. Clin. Lab. Anal. 11:186-189, 1997. @1997Wiley-Liss, Inc.

Key words: ICAM-1; ATL; HTLV-1

\section{INTRODUCTION: PROGRESSIVE T-CELL MALIGNANCY}

Adult T cell leukemia (ATL) is known as a progressive Tcell malignancy induced by an infection of human T-cell-leukemia virus type 1 (HTLV-1). Immortalizing CD4 positiveATL cells infected by HTLV-1 constitutively produce different kinds of cytokines, such as IL-1 $\alpha$, IL-1 $\beta$, TNF $\alpha$, TNF $\beta$. HTLV-1 positive human T cells express HLA class II antigen and IL-2 receptor $\alpha$ chain, which are activation antigens in T-cell differentiation.

As reported by Hinuma et al (1), the level of intercellular adhesion molecule-1 (ICAM-1) expression on ATL cells was high. Moreover, our study showed that the serum levels of soluble ICAM-1 were significantly higher in patients with ATL than in patients with myeloid leukemia and in healthy controls (2). It is of interest to analyze adhesion molecule (such as ICAM1) expression on ATL cells, sinceATL cells frequently infiltrate to the skin and invade to other organs such as liver, spleen, and lymphnode, compared with other leukemias. The present report is concerned with investigation of the factors influencing a remarkable induction of ICAM-1 molecules on ATL cells.

\section{MATERIALS AND METHODS Cell Lines}

All human cultured T-lymphoid cells and ATL cells were routinely maintained in RPMI-1640 medium supplemented with $10 \%$ fetal calf serum (FCS), $2 \mathrm{mM} \mathrm{L-glutamine} \mathrm{and}$ gentamycin sulphate $(25 \mu \mathrm{g} / \mathrm{ml})$. Molt-4 and Jurkat were all cultured human T-lymphoid cells derived from acute lymphoblastic leukemia (ALL). ILT-mat, ILT-mor, ATL16T(-), and ATL2 were cultured leukemic cells derived fromATL patients. TL-Su was derived from asymptomatic HTLV-1 carriers. Sez627c was cultured T-lymphoid cells derived from the patient of Sezary's syndrome with confirmation of infection of HTLV-1. ATL16T(-), ATL2, and Sez627c were given from Dr. Maeda (Kyoto University). ILT-mat, ILT-mor, and TL-Su were donated by Dr. Suganuma (Tohhoku University). ILTmat and ILT-mor were maintained in the presence of IL-2; others were maintained without IL-2.

\section{Northern Blot Hybridization With ICAM-1 cDNA Probe}

Northern blot analysis was carried out according to Goldberg's protocol (3). In brief, total RNAs (10 $\mu \mathrm{g}$ each) prepared from cultured T-lymphoid cells and ATL cells were fractionated by electrophoresis on gel containing formaldehyde. After electrophoresis, the gel was blotted to a filter

*Correspondence to: Masayoki Tsujisaki, South1 West16, Chuo-ku, Sapporo 060, Japan.

Received 9 August 1996; Accepted 26 November 1996 
membrane. Hybridization was carried out with human ICAM-1 cDNA probe with ${ }^{32} \mathrm{P}$-labelled using Nick translation methods (4). After washing, the filter membrane was exposed to an X-ray film at $-80^{\circ} \mathrm{C}$ with an intensifying screen.

ICAM-1 cDNA probe was synthethized by reverse transcriptase-polymerase chain reaction (RT-PCR) method. The primers were constructed from the information available on the conserved sequence of the binding site to LFA-1 of ICAM-1, following the sense primer: 5'-TGATGGGCAGTCAACAGCTA-3', the antisense primer: 5'-GAAGGATCGTTGCCATAGG-3’ (5). Human X chromosome-encoded PGK (phosphoglycerate kinase) probe was used as control probe (6).

\section{Monoclonal Antibodies}

MoAb HA58 (IgG1) to the binding site of ICAM-1 was developed in our laboratory (7). The MoAb was purified from ascitic fluid either by affinity chromatography on protein ASepharose, by iron exchange chromatography on DEAE or caprylic acid precipitation (8).

\section{Direct Cell Binding Assay}

The direct binding assay was performed as follows: $50 \mu \mathrm{l}$ of ${ }^{125}$ I-labelled MoAb HA58 $\left(1 \times 10^{5} \mathrm{cpm} /\right.$ well $)$ was incubated in a 96-well U-bottom flexible assay plate (Falcon 3911, Beckton Dickinson) with the culture cells $\left(3 \times 10^{5}\right)$ at $4{ }^{\circ} \mathrm{C}$ for $120 \mathrm{~min}$. After five washings, the wells were cut and the radioactivity was counted in a $\gamma$-counter.

\section{ATL Patient With Skin Eruption}

A 50-year-old female, who presented with leukocytosis, bilateral cervical, and inguinal lymphadenopathy and skin eruption, was admitted to our hospital in March 1994. Surface marker analysis of peripheral lymphocytes revealed $49.5 \%$ CD3 + cells, 93.4\% CD4+, 1.0\% CD8+, 76.2\% CD25 (IL-2-receptor)+, 14.7\% HLA-DR+ and 1.4\% ICAM-1+. HTLV-1 antibody was positive. Southern blot analysis using the peripheral lymphocytes showed the monoclonal integration of HTLV-1 proviral band by digestion of high molecular weight DNAs with EcoRI $\sim 9.0 \mathrm{~kb}$. According to these results, the patient was diagnosed with ATL. Skin biopsy of vesiculobullous eruption was performed in the dorsal aspect of a hand. In order to study the phenotype of invasive ATL cells to a skin, a conventional procedure for the immunoperoxidase technique was employed for the staining reactions with the antibodies.

\section{RESULTS AND DISCUSSION}

To compare the expression level of ICAM-1 mRNAs with that of cell surface ICAM-1 molecules, Northern blot hybridization was done. Two T-lymphoid cells and six ATL cells were examined for the expression of ICAM-1 mRNAs (3.3 $\mathrm{kb})$. As shown in Figure 1, the levels of ICAM-1 mRNA ex-

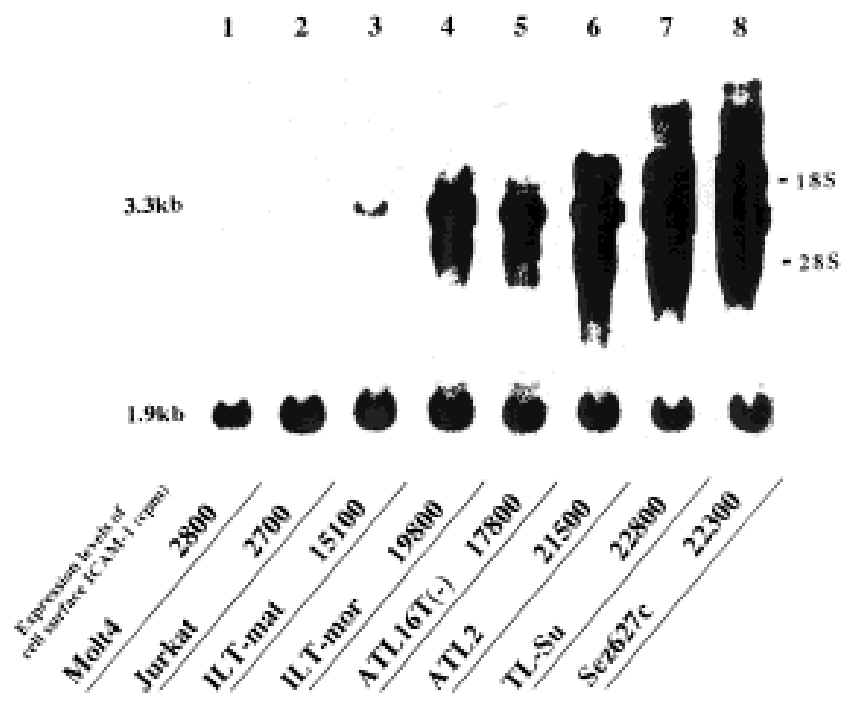

Fig. 1. Expression of ICAM-1 mRNAs ( $3.3 \mathrm{~kb})$ and cell surface ICAM-1 antigens. Human X chromosome-encoded phosphoglycerate kinase (PGK) probe was used as a control probe (PGK mRNA: $1.9 \mathrm{~kb}$ ). Expression of cell surface ICAM-1 antigens are expressed as bound cpm. The results of Northern blot hybridization corresponded to that of surface ICAM-1 expression.

\section{$\begin{array}{lllllllll}1 & 2 & 3 & 4 & 5 & 6 & 7 & 8 & 9\end{array}$}

23.1-

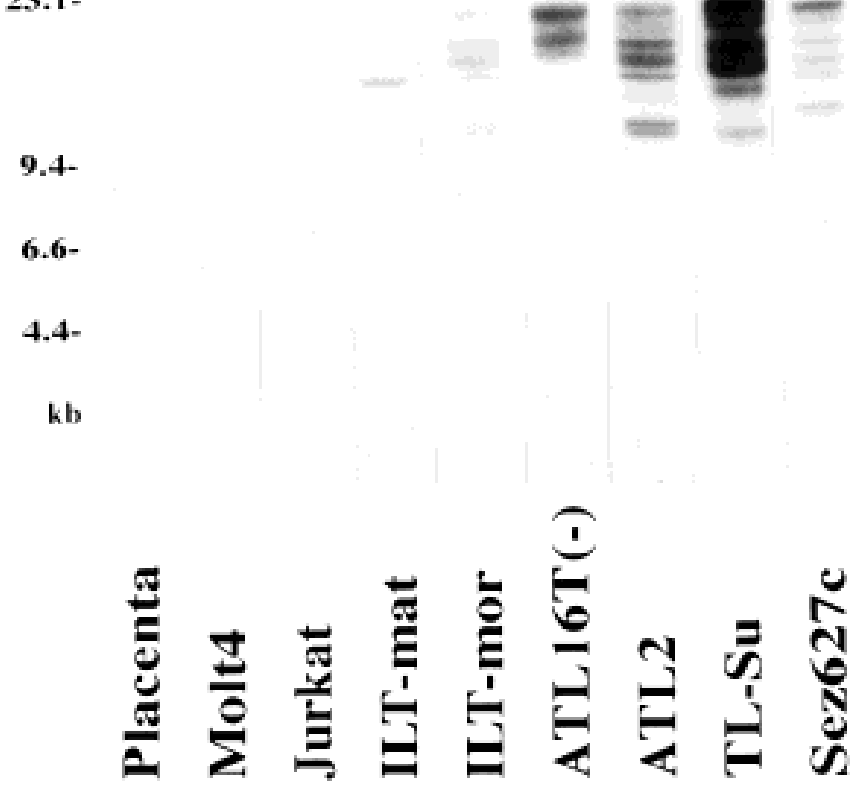

Fig. 2. Southern blot analysis of $T$ cells and ATL cells. Integration of HTLV-1 provirus was analyzed by Southern blot hybridization. DNA prepared from human placenta (lane 1) was used as a negative control. Tlymphoid cells (lanes 2 and 3) expectedly showed no band at all, whereas some bands were detected in ATL cells (lanes 4-9). 
pression of ATL cells (ILT-mor, ATL16T(-), ATL2, TL-Su, and Sez627c) were generally higher than those of T-lymphoid cells (Molt-4 and Jurkat). However, ICAM-1 mRNA expression of ILT-mat, which is one of the cultured ATL cells, was relatively lower than that of other ATL cells. The results of Northern blot hybridization corresponded to that of surface ICAM-1 expression (Fig. 1). It is of interest that ILT-mat cells, in spite of ATL cells, showed low surface ICAM-1 expression and also low ICAM-1 mRNA expression. In addition, the levels of ICAM-1 mRNA and surface ICAM-1 expression of ATL16T(-) cells were intermediate.

Cultured ATL cells are known to produce a lot of cytokines, such as IL-1 $\alpha$, TNF $\beta$, and IFN, which have inducible effects for ICAM-1 expression. The level of autocrine cytokines in the spent media of ATL cells was measured to see whether the induction of ICAM-1 expression depended on these secreted cytokines or not. However, there was no significant correlation between the levels of ICAM-1 expression and those of autocrine cytokines (data not shown).

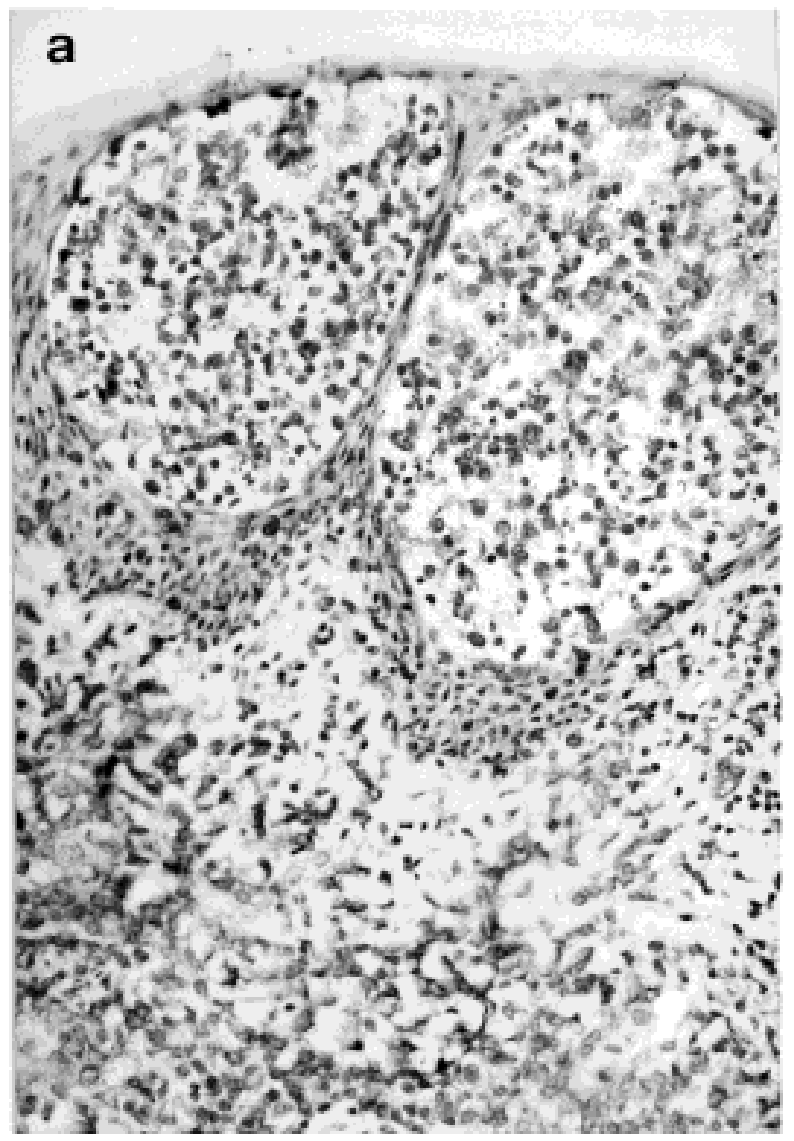

Fig. 3. Immunohistochemical features of the lymphoid cells in the vesiculobullous lesion (Original magnification $\times 170$ ): (a) anti-CD25 (IL2receptor) antibody, (b) anti-ICAM-1 MoAb HA58. CD25 positive ATL
Next, the integration of HTLV-1 provirus was analyzed by Southern blot hybridization in six cultured ATL cells and two T-lymphoid cells. DNAs (10 $\mu \mathrm{g}$ each) prepared from cultured cells with the treatment of EcoR I were fractionated by electrophoresis on $0.7 \%$ agalose gel, then blotted to a filtermembrane. According to the Nick translation method, hybridization was carried out with HTLV-1 probe pHT(M)3.9 (generous gift from Dr. Shimotohno, Kyoto University), which recognizes a part of pol, env, pX, and a part of 3'LTR of HTLV-1.

Since there is no EcoR I site in the genomes of HTLV-1 provirus, each band implys the monoclonal integration of at least one HTLV-1 genome including deletion fragments. If more than two copies of HTLV-1 genomes are integrated between each EcoR I site, an intensity of band will be strong. Therefore, both the number and the intensity of each band signify the copy amounts of HTLV-1 genomes. As shown in Figure 2, two and four bands were found in ILT-mat and ATL16T(-), respectively, whereas $>10$ bands were detected in other ATL cells (ILT-mor, ATL2, TL-Su, and Sez627c). T-

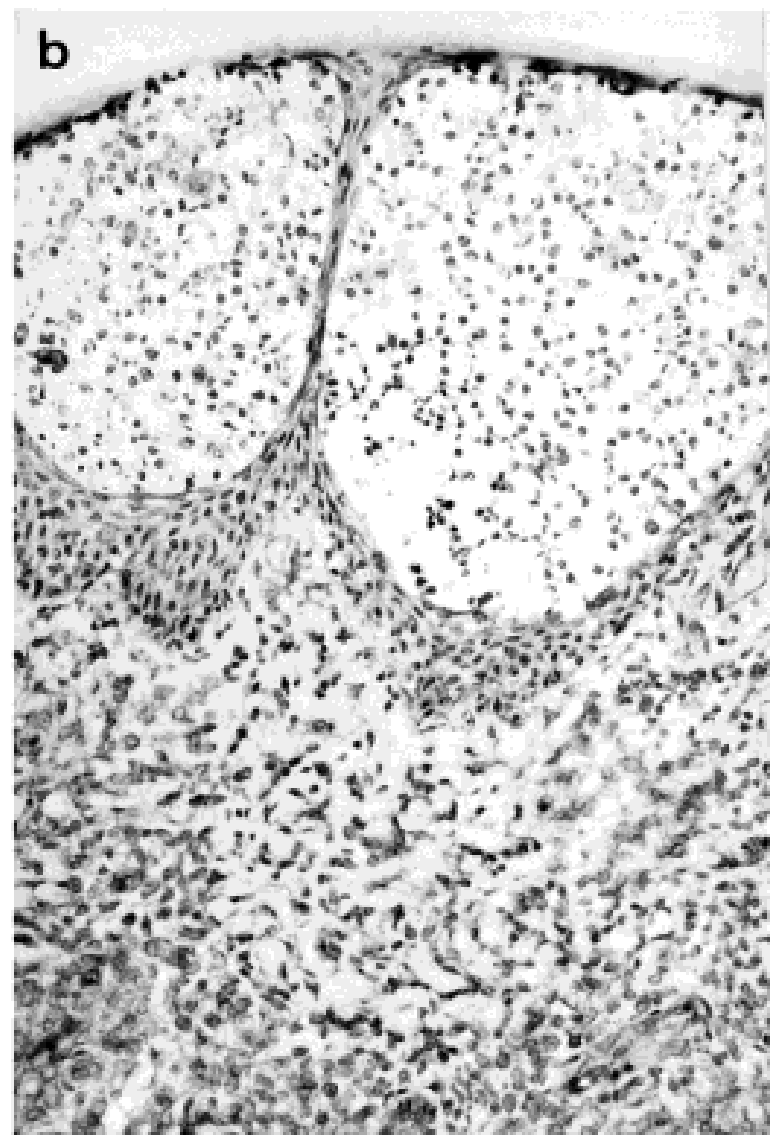

cells were widely distributed in blisters (vesicles), dermis, and epidermis. In contrast, ICAM-1 positive ATL cells preferentially infiltrated only to the dermis. 
lymphoid cells (Jurkat and Molt-4) expectedly showed no band at all.

As described above, ICAM-1 expression levels of ILT-mat and ATL16T(-) cells were lower than those of other ATL cells. These results may suggest that monoclonal integration of HTLV-1 provirus to the genome of T cell, especially the number of integration sites is one of the factors for induction of ICAM-1 molecules. It has known that leukemiogenic transacting function based on nonspecific integration site of proviruses in ATL (9). In addition, Ballard et al. (10) reported that HTLV-1 tax-induced cellular protein, which activated the NF$\mathrm{kB}$ element in the IL-2 receptor $\alpha$ gene. Sequence analysis of ICAM-1 genomic clone indicated that an NF-kB consensus element (GGGGGATTCCC) (11) was found in the upstream sequence of ICAM-1 gene (GGAGGATTCCT) (12). Therefore, it may be assumed that monoclonal integration of HTLV-1 provirus into multiple sites of T-cell genome contributes to induction of ICAM-1 expression by utilizing NF-kB like factor and its binding sites.

It is still uncertain about the mechanism of remarkable induction of ICAM-1 by HTLV-1 integration, so further analysis will be required. However, ICAM- 1 molecules expressed on ATL cells must have an important role of frequent skin infiltration, lymphadenopathy, and organ invason (hepatomegaly and splenomegaly), which are characteristics to ATL. Our study showed that the level of ICAM-1 antigens on ATL cells in the invasive skin lesion of ATL patients was much higher than that in peripheral blood. The immunohistochemical features of ATL cells in the vesiculobullous lesions was shown in Figure 3. ATL cells infiltrating to the dermis were small to medium size pleomorphic cells, whereas those infiltrating to the epidermis were large. Most of the ATL cells in the dermis expressed ICAM-1 and HLA-DR, but those in the epidermis did not. These observation suggested that preferential infiltration of ICAM-1 positive ATL cells to dermis and negative cells to epidermis might induce the formation of skin eruptions. Furthermore, the level of soluble ICAM-1 in sera of ATL patients with hepatosplenomegaly (135.5 \pm
70.1 unit) was higher than that without hepatosplenomegaly $(62.7 \pm 34.7$ unit). The study of adhesion molecules in ATL cells will be interesting to understand the feature of ATL.

\section{REFERENCES}

1. Fukudome K, Furuse M, Fukuhara N, Orita T, Hinuma Y: Strong induction of ICAM-1 in human T cells transformed by human T-cell leukemia virus type 1 and depression of ICAM-1 or LFA-1 in adult T-cell leukemia-derived cell lines. Int J Cancer 52:418-427, 1992.

2. Imai K, Nakano T, Motoya S, Tsujisaki M, Hinoda Y, Yachi A: Increased circulating intercellular adhesion molecule-1 in adult $\mathrm{T}$ cell leukemia patients. Int J Hematol 56:239-240, 1992.

3. Goldberg DA: Isolation and partial characterization of Drosophilia alcohol dehydrogenase gene. Proc Natl Acad Sci USA 77:5794-5798, 1980.

4. Rigby PWJ, Dieckmann M, Rhodes C, Berg P: Labeling deoxyribonucleic acids to high specific activity in vitro by nick translation with DNA polymerase I. J Mol Biol 113:237-251, 1977.

5. Stanton DE, Marlin SD, Stratowa C, Dustin MI, Springer TA: Primary structure of ICAM-1 demonstrates interaction between members of the immunoglobulin and integrin supergene families. Cell 52:925-933, 1988.

6. Michelson AM, Markham AF, Orkin SH: Isolation and DNA sequence of a full length cDNA clone for human $\mathrm{X}$ chromosome encoded phosphoglycerate kinase. Proc Natl Acad Sci USA 80:472-476, 1983.

7. Tsujisaki M, Imai K, Hirata H, Hanzawa Y, Masuya J, Nakano T, Sugiyama T, Matsui M, Hinoda Y, Yachi A: Detection of circulating intercellular adhesion molecule-1 antigen in malignant disease. Clin Exp Immunol 85:3-8, 1991.

8. Russo C, Callegaro L, Lanza E, Ferrone S: Purification of IgG monoclonal antibody by caprylic acid precipitation. J Immunol Meth 65:269271, 1983.

9. Seiki M, Eddy R, Shows TB, Yoshida M: Non-specific integration of HTLV provirus genome into adult T-cell leukemia cells. Nature 309:640642, 1984.

10. Ballard DW, Bohnlein E, Lowenthal JW: HTLV-1 tax induces cellular proteins that activate the $\mathrm{kB}$ elements in the IL-2 receptor $\alpha$ gene. Science 241:1652-1655, 988.

11. Lenardo MJ, Baltimore D: NF-kB: A pleiotropic mediator of inducible and tissue-specific gene control. Cell 58:227-229, 1989.

12. Wawryc SO, Cockerill PN, Wicks IP, Boyd AW: Isolation and characterization of the promoter region of the human intercellular adhesion molecule-1 gene. Int Immunol 3:83-93, 1990. 